\title{
EL ANÁLISIS DE LA INTERPRETACIÓN Y LA INTERPRETACIÓN DEL ANÁLISIS
}

Ana Llorens
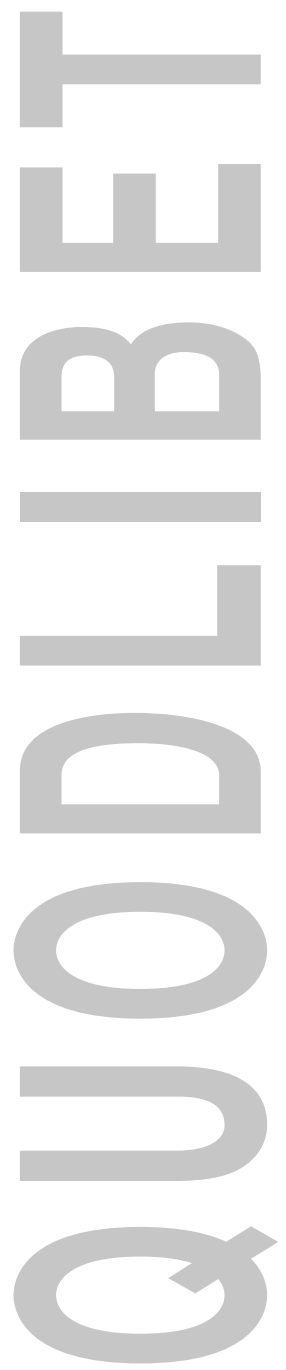

${ }^{1}$ Varios proyectos que investigaron el legado musical grabado surgieron a comienzos del siglo xxi, tales como los que convergieron en el Centro para la historia y el Análisis de la Música Grabada, 2004-2009, una iniciativa conjunta de las universidades de Royal Holloway, Londres y Sheffield. De un modo similar, la Sociedad Americana de Teoría Musical (SMT) cuenta con un grupo de estudio centrado en el análisis de la interpretación. Además, en varias universidades del ámbito anglosajón los investigadores y docentes pueden desarrollar sus carreras en esta dirección. El congreso de la Red Internacional de Estudios de la Interpretación nació con la intención de ser el punto de encuentro de académicos y músicos interesados en el estudio de la interpretación musical desde varios ángulos, no solo desde la perspectiva del análisis y la teoría.

${ }^{2}$ John Rink, «The estate of play in performance studies», en The Music Practitioner: Research for the Music Performer, Teacher and Listener, ed. por Jane W. Davidson, 37-52 (Aldershot: Ashgate, 2004), 37.

${ }^{3}$ A pesar de los avances que he mencionado en la nota 1, existen muy pocas revistas especializadas en la materia, Music Performance Research todavía está activa, pero desde 2013 solo publica un volumen cada dos años.

Recepción: 06-07-2021. Aceptación: 26-08-2021. 
desde el punto de vista teórico la comunidad musicológica ha empezado a aceptar a la interpretación como un objeto de estudio digno, institucionalmente aún se perciben ciertas reticencias.

Dentro del campo de los estudios de la interpretación, y al margen de las investigaciones sobre las prácticas interpretativas históricas y la realidad psicológica del quehacer musical, el análisis de la interpretación ha experimentado profundos cambios desde el final de los años 80 del siglo pasado. En unos años críticos, varios estudiosos alzaron sus voces para reivindicar la aceptación de la interpretación dentro del análisis y la teoría musical ${ }^{4}$. Estas reivindicaciones se desarrollaron de la mano con los retos teóricos que se plantearon a la idea de la «obra» musical como unívocamente transmitida en las partituras de los compositores ${ }^{5}$. Esto hizo que, conceptualmente, el intérprete pasara de ser un «intermediario» entre compositor y audiencia a convertirse en un co-creador de pleno derecho ${ }^{6}$. Como el único modo de preservación del sonido y planteando importantes retos ontológicos, las grabaciones fueron cosificadas como los nuevos objetos de investigación ${ }^{7}$; la literatura sobre el análisis, principalmente cuantitativo, de las interpretaciones y el software para la extracción de datos florecieron a comienzos del milenio. Sin embargo, con algunas excepciones notables en la práctica no se aceptó totalmente la dependencia temporal y contextual inherente a la interpretación de la música; no se efectuó un verdadero cambio de paradigma sino que cambiamos un objeto escrito por uno grabado ${ }^{8}$. Por otra parte, ahora que el compositor y el intérprete ocupaban unas posiciones más equilibradas,

${ }^{4}$ En este sentido se hace necesario mencionar una serie de artículos que se publicaron en aquellos años y que podemos considerar como fundacionales para la disciplina. Véanse, por ejemplo, Janet Schmalfeldt, «On the relation of analysis to performance: Beethoven’s “Bagatelles” pp. 126, nos. 2 and 5», Journal of Music Theory 19, n. 1 (1985): 1-31; Eugene Narmour, «On the relationship of analytical theory to performance and interpretation», en Explorations in Music, the Arts, and Ideas: Essays in Honor of Leonard B. Meyer, ed. por Eugene Narmour y Ruth A. Solie, 317-340 (Stuyvesant, NY: Pendragon Press, 1989); Jonathan Dunsby, «Guest editorial: Performance and analysis of music», Music Analysis 8, n. ${ }^{\text {os } 1-2 ~(1989): ~ 5-20 ; ~ y ~ l a ~ r e s e n ̃ a ~ d e ~ J o h n ~ R i n k ~ s o b r e ~ M u s i c a l ~ S t r u c t u r e ~ a n d ~ P e r f o r m a n c e ~ d e ~ W a l l a c e ~ B e r r y ~(N e w ~ H a v e n: ~ Y a l e ~}$ University Press, 1989), publicada en Music Analysis 9, n. 3 (1990): 319-339.

${ }^{5}$ Para una interpretación reciente de la idea de la «obra» musical, véase Gavin Steingo, «The musical work reconsidered, in hindsight», Current Musicology 97 (2014): 81-112.

${ }^{6}$ Para una reflexión profunda sobre este tema, véase Nicholas Cook, «Between process and product: Music and/as performance», Music Theory Online 7, n. ${ }^{\circ} 2$ (2011): 6, https://mtosmt.org/issues/mto.01.7.2/mto.01.7.2.cook.html.

${ }^{7} \mathrm{Sin}$ duda fueron fundamentales una serie de libros publicados por Cambridge University Press: The Practice of Performance: Studies in Musical Interpretation, ed. por john Rink (Cambridge: Cambridge University Press, 2015); Musical Performance: A Guide to Understanding, ed. por John Rink (Cambridge: Cambridge University Press, 2002); y The Cambridge Companion to Recorded Music, ed. por Nicholas Cook, Eric Clarke, Daniel Leech-Wilkinson y John Rink (Cambridge: Cambridge University Press, 2009). Del mismo modo, The Changing Sound of Music fue la primera monografía dedicada al análisis de las interpretaciones musicales grabadas; véase Daniel Leech-Wilkinson, The Changing Sound of Music: Approaches to Studying Recorded Musical Performance (Londres: CHARM, 2009), http://www.charm.rhul.ac.uk/studies / chapters/intro.html.

${ }^{8}$ Véase Cook, «Between process and product...». 
frecuentemente fue el analista el que fue situado en un puesto privilegiado con respecto al músico y, por tanto, no se consiguió una verdadera «superposición» entre sus esferas de acción ${ }^{9}$.

Dentro de los márgenes formales del discurso académico, recientemente se han planteado nuevas perspectivas acerca del diálogo o la relación entre interpretación y análisis, y nos han advertido sobre los peligros de la cuantificación vacía, buscando en cambio explorar la naturaleza dinámica y variable de la interpretación musical ${ }^{10}$. Aunque algunos han optado por explorar caminos más amplios y así estudiar la música como una práctica artística holística que incluso acepta la exploración autoetnográfica $^{11}$, los artículos incluidos en este monográfico plantean nuevas perspectivas en el análisis de la interpretación grabada de la música culta occidental. Dicho de otro modo, aunque en algunos círculos académicos se puede apreciar un cierto interés en la música como práctica artística en sentido amplio, muchos, entre los que nos encontramos los autores de este monográfico, creemos que aún queda mucho que decir en el campo del análisis de la interpretación. Dialogamos de tal modo que cada uno de nosotros analiza grabaciones desde un ángulo diferente y para diversos propósitos ${ }^{12}$. Mientras que las tres primeras contribuciones, de Daniel Barolsky, Adam Behan y Marco Fatichenti, abordan cuestiones conceptuales acerca del lenguaje que empleamos en el análisis de la interpretación, la función de la música en la vida de un intérprete y la idea de canon interpretativo, Jonathan Dunsby, Yannis Rammos y yo recurrimos a métodos cuantitativos para valorar micro prácticas interpretativas referentes a la asincronía y la afinación no temperada.

${ }^{9}$ Tomo prestada la expresión de Jonathan Dunsby en su «Guest editorial...», 14.

${ }^{10}$ En el momento de su publicación, el libro de Nicholas Cook Beyond the Score marcó una piedra angular en los estudios de la interpretación musical y en su análisis en particular; véase Nicholas Cook, Beyond the Score: Music as Performance (Nueva York: Oxford University Press, 2013).

${ }^{11}$ Véanse, por ejemplo, The Music Practitioner: Research for the Music Performer, Teacher and Listener, ed. por Jane W. Davidson (Aldershot: Ashgate, 2004); Expressiveness in Music Performance: Empirical Approaches across Styles and Cultures, ed. por Dorottya Fabian, Renée Timmers y Emery Schubert (Oxford: Oxford University Press, 2014); Artistic Practice as Research in Music: Theory, Criticism, Practice, ed. por Mine Doğantan-Dack (Aldershot: Ashgate, 2015); y Musicians in the Making: Pathways to Creative Performance, ed. por John Rink, Helena Gaunt y Aaron Williamon (Nueva York: Oxford University Press, 2017). Notablemente, los tres últimos títulos surgieron dentro del CMPCP, el Centro para la Interpretación Musical como Práctica Creativa, con sede en la Universidad de Cambridge y dirigido por John Rink entre 2009 y 2014. Hasta cierto punto, el CMPCP fue la continuación del CHARM (véase la nota 1), siendo ejemplo del cambio desde el análisis puro a un enfoque más amplio. En España, hemos tenido que esperar hasta el año pasado para ser testigos de la publicación del primer volumen monográfico sobre investigación artística en música, precisamente en Quodlibet (n. $\left.{ }^{\circ} 74\right)$.

${ }^{12}$ Como ejemplos del aún floreciente análisis de la interpretación musical, véanse John Rink, «The (f)utility of performance analysis», en Artistic Practice as Research in Music: Theory, Criticism, Practice, ed. por Mine Doğantan-Dack, 127147 (Aldershot: Ashgate, 2015); Jeffrey Swinkin, Performative Analysisr Reimagining Music Theory for Performance (Rochester: University of Rochester Press, 2016); y Daphne Leong, Performing Knowledge: 20th Century Music in Analysis \& Performance (Nueva York: Oxford University Press, 2019). 
Daniel Barolsky abre este monográfico poniendo sobre la mesa el diálogo — lingüísticoque se establece entre composición e interpretación, que relaciona con la posición tradicionalmente privilegiada de compositores y analistas en relación al intérprete, como he comentado más arriba. Analizando la grabación de Ernst Levy de las variaciones de Brahms sobre un tema de Haendel, Barolsky propone una nueva manera de aceptar que las interpretaciones pueden crear experiencias estéticas que no tienen por qué proyectar nada contenido en la partitura de un compositor, ya sea de forma tácita o explícita. En última instancia, nos impulsa a estudiar y a analizar las interpretaciones musicales como eventos únicos, evitando así los prejuicios inherentes a compararlas con otras grabaciones o análisis previos.

En su artículo, Adam Behan analiza el cambio interpretativo que se produjo en Glenn Gould cuando en 1964 el pianista decidió alejarse de las salas de concierto para centrarse en las salas de grabación. A través de seis escenas que «se superponen» y que exploran seis facetas de la carrera de Gould —como intérprete en el escenario y en el estudio de grabación, como entrevistado y como escritor de ensayos, y delante de las cámaras fotográficas y de televisión-, Behan reflexiona acerca del modo en el que la interpretación y las actividades alrededor de ella pueden convertirse en actos de cuidado personal. Dando un paso más allá, el formato de las seis escenas reproduce el del ensayo del propio Gould titulado «Stokowski en seis escenas», presentándonos al artista consciente desde una perspectiva interna.

Por su parte, Marco Fatichenti profundiza en la relación entre interpretación, composición y estética musical al explorar un tema profundamente arraigado en esta última —el nacionalismono a través del prisma habitual de la segunda sino desde la perspectiva de la primera. En concreto, analiza la práctica interpretativa en torno a Goyescas de Enrique Granados y explora los modos en que el clima ideológico pudieron haberse traducido en un canon interpretativo bien definido tanto en España como en el extranjero. De este modo, Fatichenti establece una colaboración íntima entre análisis e interpretación, proponiendo en última instancia una visión interpretativa renovada de El amory la muerte.

Para cerrar este monográfico, las visiones acerca de la interpretación musical se alejan de consideraciones más filosóficas y estéticas y se centran en las peculiaridades interpretativas a pequeña escala. Jonathan Dunsby y Yannis Rammos posan su mirada sobre la asincronía melódica en la música culta occidental. Su trabajo reconoce la realidad de la creatividad física y corpórea analizando continuidades sonoras y diversos tipos de asincronía en un rollo en el que el propio Debussy interpreta su «The little shepherd». Situando a la asincronía en el contexto de la evidencia histórica y teórica, los autores reevalúan ideas de audibilidad e intencionalidad en la percepción y en la interpretación de la música. 
Como Dunsby y Rammos, en el ultimo trabajo de este monográfico recurro a métodos cuantitativos de análisis, en este caso para evaluar las estrategias de afinación de Pau Casals en su grabación del preludio de la Suite n. ${ }^{\circ} 4$ para violonchelo solo de Bach. Basándome en un análisis sistemático de los datos desde varios ángulos teóricos plausibles, muestro cómo la «afinación expresiva» de Casals puede adquirir un nuevo significado en el contexto de sus demás estrategias interpretativas, puesto que todas ellas se combinan para darle forma a la pieza como una sucesión de momentos de tensión y relajación.

A través del análisis de ejemplos grabados de nuestro patrimonio musical, los cinco trabajos contenidos en este monográfico suponen un reto para las jerarquías tradicionalmente establecidas dentro de la musicología e incluso dentro del análisis de la interpretación, situando todas ellas a la interpretación en el centro del estudio analítico y revisando ideas tradicionales acerca de la interpretación y el estilo interpretativo. En última instancia, ilustran nuevas formas en las que teoría, análisis e interpretación pueden hacerse justicia mutuamente, en la búsqueda por una verdadera simbiosis entre los dos campos. Siendo estas las primeras páginas dedicadas al análisis de la interpretación musical en una revista española, esperamos que sirvan para impulsar investigaciones posteriores en estas u otras líneas. 